\title{
Fabrication of Tin Oxide Thin Film Transistors by RF Magnetron Sputtering Using Sn/SnO Composite Target
}

\author{
Cheol Kim, Sarah Eunkyung Kim* \\ Graduate School of Nano-IT Design, Seoul National University of Science and Technology, Seoul, Korea
}

Email address:

eunkyung@seoultech.ac.kr (S. E. Kim)

${ }^{*}$ Corresponding author

To cite this article:

Cheol Kim, Sarah Eunkyung Kim. Fabrication of Tin Oxide Thin Film Transistors by RF Magnetron Sputtering Using Sn/SnO Composite Target. Advances in Materials. Vol. 7, No. 3, 2018, pp. 73-77. doi: 10.11648/j.am.20180703.13

Received: July 12, 2018; Accepted: July 24, 2018; Published: August 17, 2018

\begin{abstract}
P-type tin oxide thin film deposited by RF sputtering for transparent thin film transistor (TFT) applications is the subject of this study. P-type tin oxide thin film can be made by doping a cation with a lower valence into n-type $\mathrm{SnO}_{2}$ as an acceptor impurity or by fabrication with tin monoxide (SnO). The later method was investigated in this study by RF magnetron sputtering process, which has a high deposition rate, uniform thickness control, simple stoichiometry control, and reproducibility. A $\mathrm{Sn} / \mathrm{SnO}$ composite target was used in $\mathrm{RF}$ sputtering to utilize the benefits of both metallic and ceramic sputtering targets. A $\mathrm{Sn} / \mathrm{SnO}$ composite target is expected to provide p-type tin oxide thin films that are easier to manufacture than metallic target or ceramic target. The metallic Sn element provides an excellent control of structural defects, while the ceramic $\mathrm{SnO}$ element provides stable stoichiometry. The p-type tin oxide thin film with a $\mathrm{Sn} / \mathrm{SnO}$ composite target showed very good transparency of $\sim 95 \%$. and excellent electrical properties. The p-type tin oxide thin film of $15 \mathrm{~nm}$ thickness had a carrier concentration of $8.03 \times 10^{15} \mathrm{~cm}^{-3}$ and a mobility of $15.2 \mathrm{~cm}^{2} / \mathrm{Vs}$. With these ultrathin tin oxide films, a p-channel TFT was fabricated with a staggered bottom-gate structure, and the effect of different channel thicknesses and different distances between the two electrodes were evaluated. The tin oxide TFT with a thick p-channel layer showed a significant increase in current measured than the tin oxide with a thin p-channel layer. The current measured tended to increase greatly as the distance between electrodes decreased. The IV output curves of the tin oxide TFTs exhibited characteristics of bipolar transistors potentially due to the partial creation of the oxygen-deficient $\mathrm{SnO}_{2}$ like structure, but the mechanism of bipolar transistor characteristics from the p-channel tin oxide TFT is still unclear. The fabrication of highly transparent tin oxide thin film with bipolar transistor characteristics is demonstrated herein.
\end{abstract}

Keywords: Tin Oxide, Sputtering, Composite Target, Thin Film Transistor, Bipolar

\section{Introduction}

Oxide semiconductors have good electrical conductivity and excellent optical transparency for use as transparent conducting materials [1]. Oxide semiconductors with higher carrier mobility are generally n-type semiconductors such as $\mathrm{In}_{2} \mathrm{O}_{3}, \mathrm{ZnO}$, and $\mathrm{SnO}_{2}$. While significant progress has been made in n-channel thin film transistors (TFTs), highly purified p-type oxide semiconductors are still required for the realization of optoelectronic devices or p-channel TFTs. The first p-type oxide semiconductors reported were p-type nickel oxide ( $\mathrm{NiO})$, thin films by Sato et al. [2] and p-type copper aluminum oxide $\left(\mathrm{CuAlO}_{2}\right)$, by Kawazoe et al. in 1990 [3].
Tin oxide thin films, due to their high carrier mobility, have recently received significant attention as promising materials for TFTs; however, only a few research groups have covered tin monoxide ( $\mathrm{SnO}$ ) thin film transistors [4-9]. Tin monoxide thin films are typically known to be p-type oxide semiconductors without any doping. Unlike n-type tin dioxide $\left(\mathrm{SnO}_{2}\right)$, thin film, which is electrically and chemically stable, p-type $\mathrm{SnO}$ thin film presents technical challenges such as low temperature processing and difficulties in mass production as well as lack of reliability $[10,11]$. However, p-type $\mathrm{SnO}$ thin film is potentailly an 
excellent material, with a large hole mobility stemming from the hybridized orbitals composed of $\mathrm{Sn} 5 \mathrm{~s}^{2}$ and $\mathrm{O} 2 \mathrm{p}$ at the VBM (valence band maximum) [4, 12, 13]. In this study, the fabrication of p-type tin oxide thin films have been investigated with the purpose of achieving a p-type characteristic for $\mathrm{p}$-channel thin film transistor applications.

There are a wide range of deposition methods for tin oxide thin films: sputtering, pulsed laser deposition, chemical vapor deposition (CVD), sol-gel techniques, and spray pyrolysis [14-22]. The electrical and structural properties of tin oxide thin films can vary depending on the deposition methods. Among these methods, sputtering is most commonly used, because it has an ability to produce dense films and an easy control of film stoichiometry. In addition, sputtering may provide a relatively high deposition rate, uniform thickness, scalability, stable chemical composition (homogeneity), and reproducibility [16]. Conversely, there are possible plasma damages and inequality of film composition. Since the sputtering method is the process produced by particle emission of the target, it is very important to select a sputter target material. Metallic $\mathrm{Sn}$, ceramic $\mathrm{SnO}$, or ceramic $\mathrm{SnO}_{2}$ targets are typically used to deposit tin oxide thin films by sputtering. The advantages of a metallic Sn target are easy control of electrical properties and compositions of tin oxide thin films as well as high deposition rate, and the disadvantages are the low melting temperature of $\mathrm{Sn}$ and the fact that the p-type processing window is narrow [16]. On the other hand, a ceramic target has stable stoichiometry, but less variation of stoichiometry. There are some reports on the use of $\mathrm{Sn} / \mathrm{SnO}$ composite target $[14,15]$. or $\mathrm{Sn} / \mathrm{SnO}_{2}$ composite target [23]. A composite target can provide a potential composition of chemically stable tin oxide thin films and can easily control material properties.

In this study, tin oxide thin films were deposited by reactive $\mathrm{RF}$ magnetron sputtering with an $\mathrm{Sn} / \mathrm{SnO}$ composite target, and electrical, optical, and structural properties of tin oxide were evaluated. Then the $\mathrm{p}$-channel tin oxide TFT was fabricated on a Si substrate, and the device characteristic of the p-channel tin oxide TFT was measured.

\section{Materials and Methods}

Reactive RF magnetron sputtering with an $\mathrm{Sn} / \mathrm{SnO}$ composite target was used; this material, compared to $\mathrm{Sn}$ metallic targets or tin oxide ceramic targets, may potentially provide manufacturable p-type tin oxide thin films as well as simpler control of structural defects [14]. The $\mathrm{Sn} / \mathrm{SnO}$ composite target used was composed of $20 \mathrm{~mol} \%$ Sn metal powder. The sintering of $\mathrm{Sn} / \mathrm{SnO}$ composite target needs more careful control than that of $\mathrm{Sn} / \mathrm{SnO}_{2}$ composite target, because the $\mathrm{SnO}$ phase compared to the $\mathrm{SnO}_{2}$ phase tends to show a disproportionation reaction [15]. The metallic Sn component in the composite target provides a means of controlling the structural defects when using RF reactive sputtering; the $\mathrm{SnO}$ component provides stable stoichiometry [15]. The sputter schematic and the image of the $\mathrm{Sn} / \mathrm{SnO}$ composite target are shown in Figure 1.

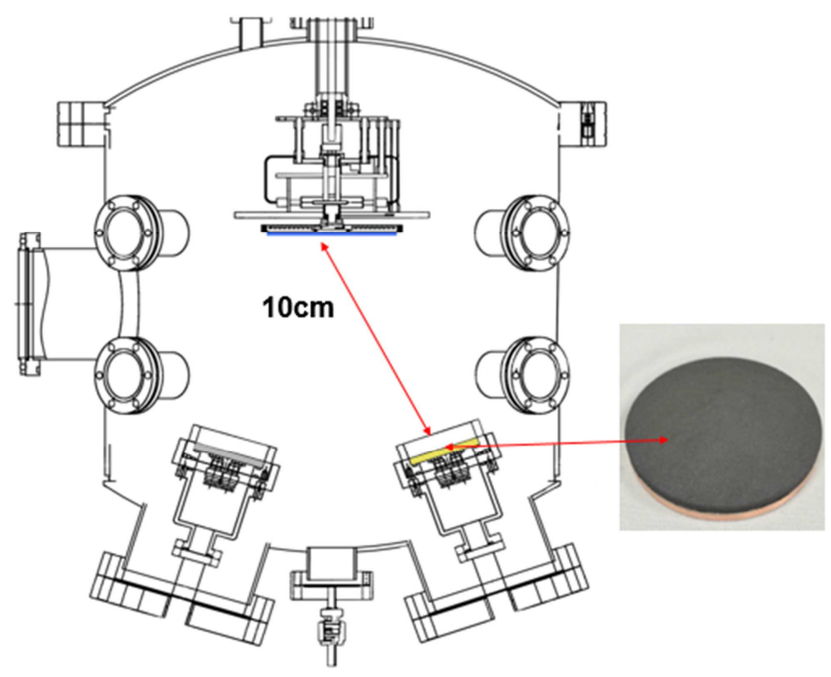

Figure 1. Sputter Schematic and Sn/SnO Composite Target.

The tin oxide thin film was deposited onto borosilicate glass. The substrate temperature was kept at $100^{\circ} \mathrm{C}$, the plasma power was set at $20 \mathrm{~W}$, and the working pressure and chamber base pressure were $5 \mathrm{mTorr}$ and $<8 \times 10^{-6}$ Torr respectively. The $\mathrm{O}_{2}$ content $(\%)$, denoted by $\mathrm{O}_{2} /\left(\mathrm{O}_{2}+\mathrm{Ar}\right)$, was $12 \%$. For the repeatability test, ten identical tin oxide thin film samples were prepared and analyzed. For the structural analysis of the tin oxide thin films, X-ray diffraction (XRD; ATX-G, Rigaku), and X-ray photoelectron spectrometry (XPS; EscaLab 250, VG Scientifics) were employed. The optical property was measured by a UV-Vis spectrometer (Lamda 35, Perkin Elmer), The electrical properties associated with the carrier concentration, carrier mobility, and electrical conductivity were measured by a Van der Pauw Hall Effect Measurement System (HMS-3000, Ecopia) operated at room temperature with a $0.55 \mathrm{~T}$ magnet, and Sn electrodes were deposited using a shadow mask.

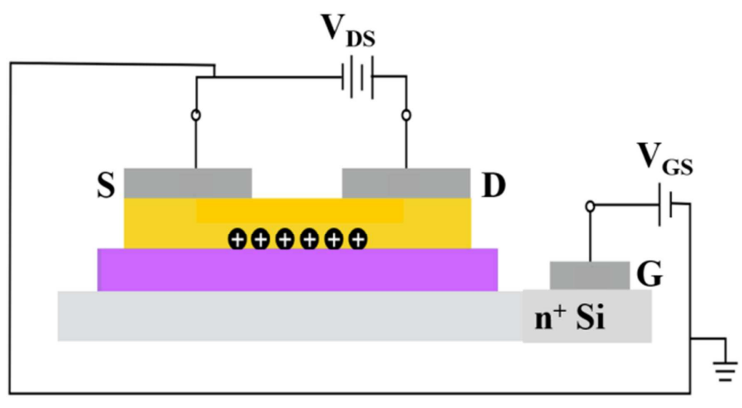

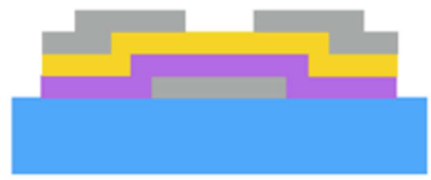

Staggered bottom gate
Substrate

Dielectric

Semiconductor

Electrode
Figure 2. Schematic Diagram of Staggered Bottom Gate Thin Film Transistor.

The tin oxide TFT fabricated in this study had a staggered bottom-gate structure, as shown in Figure 2. The fabrication flow of the TFTs is shown in Figure 3. A heavily n-doped ( $\rho$ 
$=0.0035 \Omega \mathrm{cm}), 6$-inch silicon wafer was used as a substrate that became a gate electrode. After wet cleaning of the $\mathrm{n}+\mathrm{Si}$ substrate, thermal oxidation was performed to grow a $\sim 60 \mathrm{~nm}$ $\mathrm{SiO}_{2}$ layer. Then, lithography patterning and $\mathrm{SiO}_{2}$ etching processes were employed to form an individual TFT device on the Si wafer. After that, the p-type tin oxide thin film was deposited as a p-channel layer, and then $\mathrm{Ni}$ electrodes for source, drain, and gate were deposited by shadow masks. Several p-channel tin oxide TFTs were fabricated with two different channel thicknesses $(15 \mathrm{~nm}$ and $35 \mathrm{~nm})$, and three

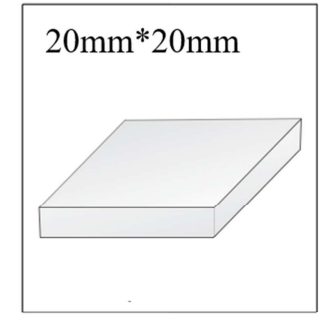

Wafer cleaning

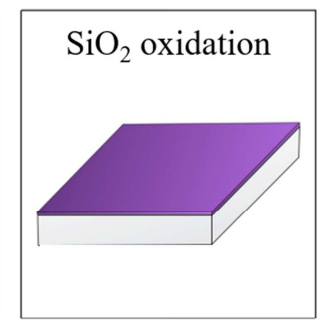

Dry oxidation

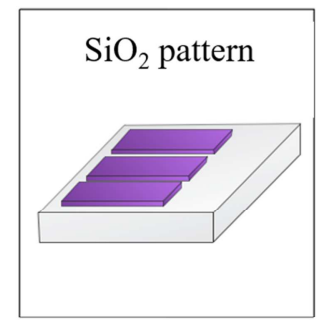

Lithography / BOE etching

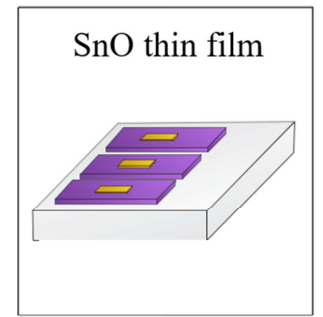

Sputter dep. (channel) different distances between the two electrodes $(100 \mu \mathrm{m}$, $500 \mu \mathrm{m}$, and $1000 \mu \mathrm{m}$ ), and the effects of tin oxide channel thickness and distance between two electrodes on TFT characteristics were evaluated. The IV characteristics of $\mathrm{p}$ channel tin oxide TFTs were measured using a semiconductor parameter analyzer (SPA; 4155C, Agilent). For the output curve $\left(\mathrm{I}_{\mathrm{DS}} / \mathrm{V}_{\mathrm{DS}}\right)$, measurements, the gate/source voltage was swept at $\pm 20 \mathrm{~V}$ (5V/step), and the source/drain voltage was swept at $\pm 20 \mathrm{~V}(0.2 \mathrm{~V} /$ step $)$.

Figure 3. Schematic Diagram of Tin Oxide Thin Film Transistor Fabrication.

\section{Results and Discussions}

According to the results of XRD analysis of the tin oxide thin film, as shown in Figure 4(a), the tin oxide thin film used in p-channel TFT fabrication appeared to be an amorphouslike phase. It potentially suggests a short-range ordered structure. Furthermore, based on the XPS analysis shown in Figure 4(b), the tin oxide thin film had a binding energy of the $\mathrm{Sn}_{3} \mathrm{~d}_{5 / 2}$ peak of $\sim 486.3 \mathrm{eV}$, indicating a p-type tin oxide; the stoichiometry of the tin oxide thin film was estimated to be $\mathrm{SnO}_{1.09}$. The electrical properties of the tin oxide thin films are presented in Table 1 which confirmed the p-type characteristics. The conductivity was on the order of $10^{-3} \Omega^{-}$ ${ }^{1} \mathrm{~cm}^{-1}$, which was sufficient for the purpose of TFT fabrication. The thinner tin oxide thin films showed lower carrier concentration and higher mobility. Additionally, all of the fabricated tin oxide thin film samples showed almost no difference in electrical properties showing a good film reproducibility. The $12 \% \quad \mathrm{O}_{2}$ content condition during sputtering adequately controlled the stoichiometry and created excellent transmittance. The tin oxide thin films exhibited good optical transmittance of about $95 \%$ in the visible range, as shown in Figure 5. (a) XRD

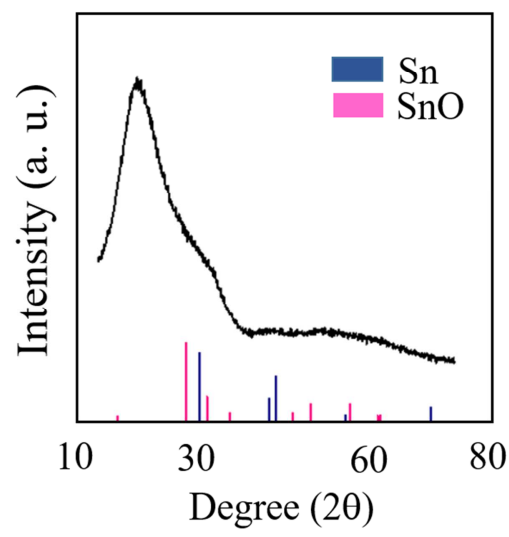

(b) XPS

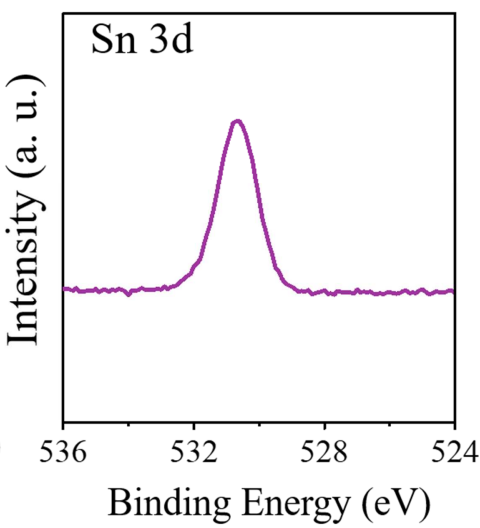

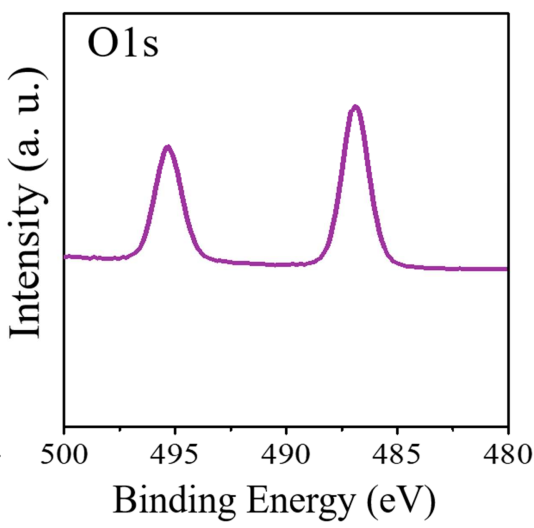

Figure 4. Structural Analysis of Tin Oxide Thin Films: (a) XRD Analysis and (b) XPS Analysis.

Table 1. Electrical Properties of P-type Tin Oxide Thin Films.

\begin{tabular}{|c|c|c|c|}
\hline SnO $_{x}$ Thickness (nm) & Carrier Concentration, $\mathrm{N}_{\text {Carrier }}\left(\mathrm{cm}^{-3}\right)$ & Mobility, $\mu\left(\mathrm{cm}^{2} / \mathrm{Vs}\right)$ & Conductivity, $\sigma\left(\Omega^{-1} \mathrm{~cm}^{-1}\right)$ \\
\hline 15 & $8.03 \times 10^{15}$ & 15.2 & $8.21 \times 10^{-3}$ \\
\hline 35 & $1.80 \times 10^{17}$ & 0.80 & $7.23 \times 10^{-3}$ \\
\hline
\end{tabular}




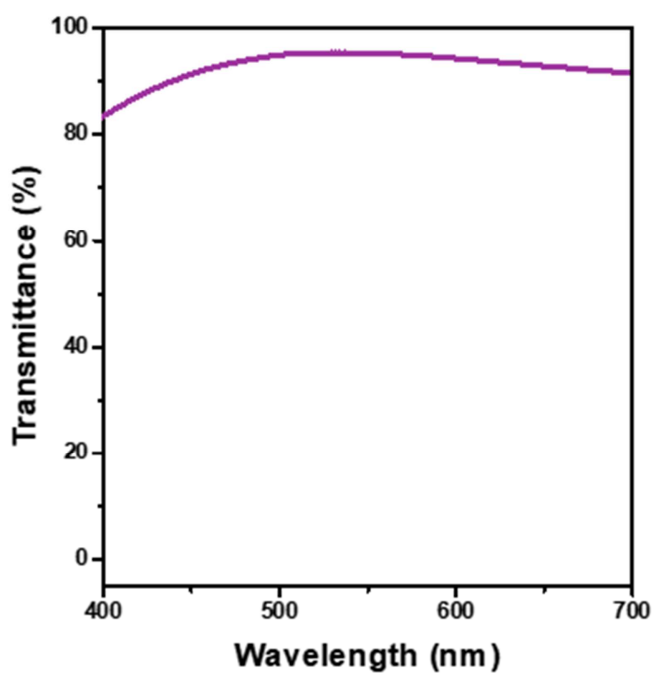

Figure 5. Transmittance Spectra of Tin Oxide Thin Film (35nm-Thick SnO Sample).

Figure 6 provides a top view of the fabricated p-channel tin oxide TFT structures. Due to the shadowing effect of the masks, edge rounding in channel and electrode patterns was observed. In general, a p-channel TFT has two regions in the IV output curve in the on-state: a linear region $\left(-\mathrm{V}_{\mathrm{DS}} \leq-\mathrm{V}_{\mathrm{GS}}\right.$ $\left.+\mathrm{V}_{\mathrm{T}}\right)$ and a saturation region $\left(-\mathrm{V}_{\mathrm{DS}} \geq-\mathrm{V}_{\mathrm{GS}}+\mathrm{V}_{\mathrm{T}}\right)$ per $\mathrm{V}_{\mathrm{DS}}$. In Figure 7 (e) and (f) the saturation region is shown somewhat, but the other cases do not reveal a clear saturation region in the sweeping voltage range. More importantly, the IV output curves in Figure 7(a) - (f) show characteristics of bipolar transistors. This means that the p-channel tin oxide TFT fabricated here was a current-controlled device, and the current flow in this device was due to both electron and hole carriers instead of a majority carrier of hole carriers. Under $12 \%$ oxygen content condition, oxygen incorporation in the p-channel tin oxide did not generate holes, but it did generate electrons, which in turn causes n-type characteristics. This may be explained by the partial creation of the oxygen- deficient $\mathrm{SnO}_{2}$ like structure.

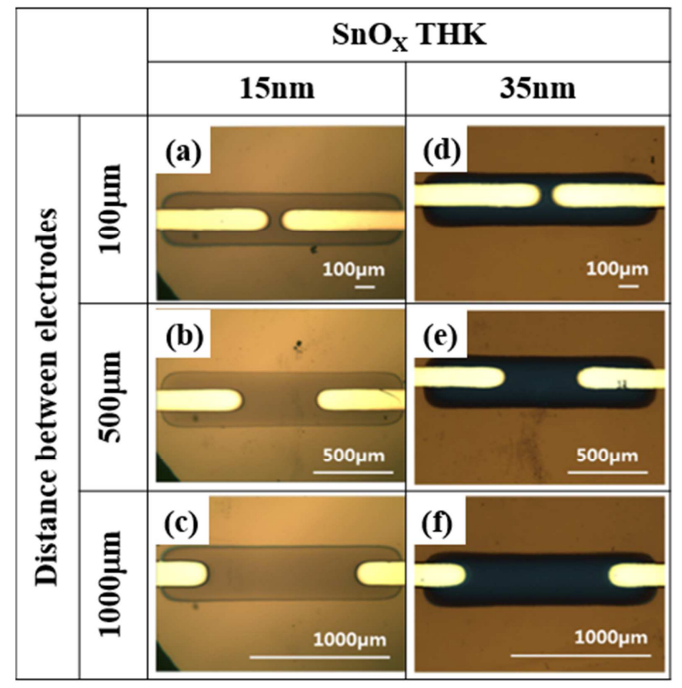

Figure 6. Optical Images of P-Channel Tin Oxide TFT.

The p-channel tin oxide TFT is operated both in accumulation $\mathrm{p}$-channel mode and inversion n-channel mode. In literature, both bipolar and unipolar transistor characteristics of p-channel tin oxide TFTs have been reported [4-12]. The measured current in the tin oxide TFT with a $35 \mathrm{~nm}$-thick channel layer was increased significantly compared to the tin oxide TFT with a $15 \mathrm{~nm}$-thick channel layer, due to a current enhancement. For the same reason, the measured current tended to increase greatly as the distance between electrodes decreased. Although the mechanism of bipolar transistor characteristics from the p-channel tin oxide TFT is still unclear, the capability of using both holes and electrons in one device opens up potential possibilities for the development of various thin film transistors and optoelectronic device.

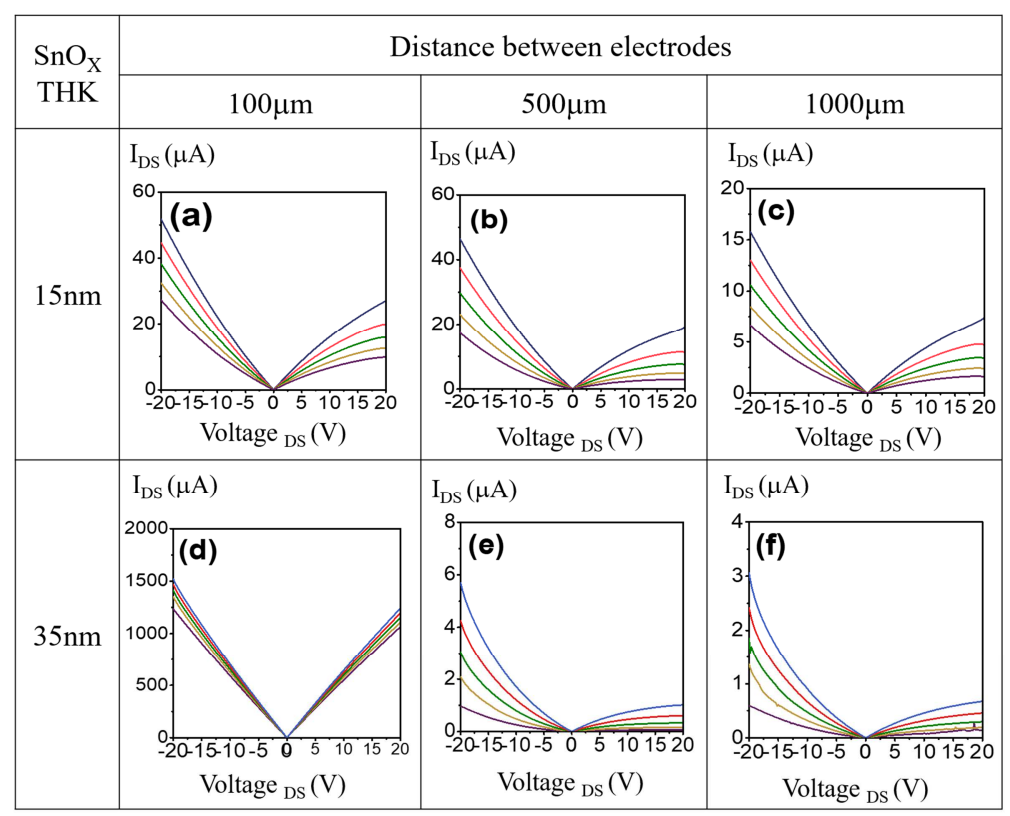

Figure 7. IV Output Curve of P-Channel Tin Oxide TFT ( $V_{G S}=-20 \mathrm{~V}$ at $\left.5 \mathrm{~V} / \mathrm{step}\right)$. 


\section{Conclusion}

P-type tin oxide is an important element for transparent thin-film transistor (TFT) applications. In this study p-type tin oxide thin films deposited using RF magnetron sputtering of $\mathrm{Sn} / \mathrm{SnO}$ composite target were studied, and films with $95 \%$ transmittance, mobility of $15.2 \mathrm{~cm}^{2} / \mathrm{Vs}$, and carrier concentration of $8.03 \times 10^{15} \mathrm{~cm}^{-3}$ were obtained. A sputtering method with a $\mathrm{Sn} / \mathrm{SnO}$ composite target provides a promising candidate for fabricating manufacturable p-type tin oxide thin films. The p-channel tin oxide TFTs fabricated in this study showed bipolar transistor characteristics, and the mechanism of bipolar transistor characteristics from p-channel tin oxide TFTs has not been elucidated.

\section{Acknowledgements}

This research was supported by the Basic Science Research Program through the National Research Foundation of Korea (NRF), funded by the Ministry of Education, Science and Technology (NRF-2015R1C1A2A01055792).

\section{References}

[1] A. N. Banerjee and K. K. Chattopadhyay, Prog. Cryst. Growth Charact. Mater. 50 (2005) 52-105.

[2] H. Sato, T. Minami, S. Takata, and T. Yamada, Thin Solid Films 236 (1993) 27-31.

[3] H. Kawazoe, M. Yasukawa, H. Hyodo, M. Kurita, H. Yanagi, and H. Hosono, Nature 389 (1997) 939-942.

[4] H. Hosono, H. T. Ogo, H. Yanagi, and T. Kamiya, Solid State Lett. 14 (2011) H13-H16.

[5] H. Luo, L. Liang, and H. Cao, Solid State Electron. 129 (2017) 88-92.

[6] P. K. Nayak, J. A. Caraveo-Frescas, Z. Wang, M. N. Hedhili, Q. X. Wang, H. N. Alshareef, Scientific Reports, 4 (2014) 4672.1-4672.7.
[7] J. A. Caraveo-Frescas, P. K. Nayak, H. A. Al-Jawhari, D. B. Granato, U. Schwingenschlog, and H. N. Alshareef, ACS Nano 7 (2013) 5160-5167.

[8] P. Hsu, W. Chen, Y. Tsai, Y. Kung, C. Chang, C. Hsu, C. Wu, and H. Hsieh, Jap. J. Appl. Phys. 52 (2013) 05DC07.

[9] H. Luo, L. Liang, H. Cao, M. Dai, Y. Lu, M. Wang, ACS Appl. Mater. Interf. 7 (2015) 17023-17031.

[10] H. Yabuta, N. Kaji, R. Hayashi, H. Kumomi, K. Nomura, T. Kamiya, M. Hirano, and H. Hosono, Appl. Phys. Lett. 97 (2010) 072111.

[11] L. Liang and H. Cao, ECS Trans. 50 (2012) 289-297.

[12] Y. Ogo, H. Hiramatsu, J. Nomura, H. Yanagi, T. Kamiya, M. Hirano, and H. Hosono, Appl. Phys. Lett. 93 (2008) 032113.

[13] J. Um and S. E. Kim, ECS Solid State Lett. 3 (2014) 94-98.

[14] C. Kim, S. Kim, and S. E. Kim, Thin Solid Films 634 (2017) 175-180.

[15] C. Kim, S. Cho, S. Kim, and S. E. Kim, ECS J. Solid State Sci Tech. 6 (2017) 765-771.

[16] S. E. Kim and M. Oliver, Met. Mater. Int. 16 (2010) 441-446.

[17] L. Roman, R. Valaski, C. Canestraro, E. Magalhaes, .C. Persson, R. Ahuja, E. da Silva Jr., I. Pepe, and A. Ferreira da Silva, Appl. Surf. Sci. 252 (2006) 5361-5364.

[18] T. Serin, N. Serin, S. Karadeniz, H. Sari, N. Tugluoglu, and O. Pakma, J. Non-Cryst. Solids 352 (2006) 209-215.

[19] T. T. Racheva and G. Q. Critchlow, Thin Solid Films 292 (1997) 299-302.

[20] J. C. Lou, M. S. Lin, K. I. Chyi, and J. H. Shieh, Thin Solid Films 106 (1993) 163-173.

[21] S. Tamura, T. Ishida, H. Magara, T. Mihara, O. Tabara, and T. Tatsuta, Thin Solid Films 343-344 (1994) 142-144.

[22] G. Liu, Z. Zhao, X. Jiao, and D. Chen, Mater. Tech. 29 (2014) 167-171.

[23] P. Hsu, S. Tsai, C. Chang, C. Hsu, W. Chen, H. Hsieh, and C. Wu, Thin Solid Films 585 (2015) 50-56. 